TITLE:

\title{
A finite element/volume method model of the depth-averaged horizontally 2D shallow water equations
}

\section{$\operatorname{AUTHOR}(S):$}

Yoshioka, Hidekazu; Unami, Koichi; Fujihara, Masayuki

\section{CITATION:}

Yoshioka, Hidekazu ... [et al]. A finite element/volume method model of the depthaveraged horizontally 2D shallow water equations. International Journal for Numerical Methods in Fluids 2014, 75(1): 23-41

ISSUE DATE:

2014-01-31

URL:

http://hdl.handle.net/2433/198579

\section{RIGHT:}

This is the peer reviewed version of the following article: Yoshioka, H., Unami, K. and Fujihara, M. (2014), A finite element/volume method model of the depth-averaged horizontally 2D shallow water equations. Int. J. Numer. Meth. Fluids, 75: 23-41, which has been published in final form at http://dx.doi.org/10.1002/fld.3882.; This is not the published version. Please cite only the published version.; この論文は出版社版でありません。引用の際には出版社版をご確認ご 利用ください。 
A finite element/volume method model of the depth averaged horizontally 2- D shallow water equations

\section{Hidekazu Yoshioka}

Research Fellow of Japan Society for the Promotion of Science, Graduate School of Agriculture, Kyoto University, Kitashirakawa-Oiwake-cho, Sakyo-ku, Kyoto 606-8502, Japan.

E-mail: yoshih@kais.kyoto-u.ac.jp (Corresponding author)

Tel: 81-75-753-6160

Fax: 81-75-753-6349

\section{Koichi Unami}

Associate professor, Graduate School of Agriculture, Kyoto University,

Kitashirakawa-Oiwake-cho, Sakyo-ku, Kyoto 606-8502, Japan.

E-mail: unami@adm.kais.kyoto-u.ac.jp

Masayuki Fujihara

Professor, Graduate School of Agriculture, Kyoto University, Kitashirakawa-Oiwake-cho, Sakyo-ku, Kyoto 606-8502, Japan.

E-mail: fujihara@adm.kais.kyoto-u.ac.jp

\section{Abstract}

Analysis of surface water flows is of central importance in understanding and predicting a wide range of water engineering issues. Dynamics of surface water is reasonably well described using the shallow water equations (SWEs) with the hydrostatic pressure assumption. The SWEs are non-linear hyperbolic partial differential equations that are in general required to be solved numerically. Application of a simple and efficient numerical model is desirable for solving the SWEs in practical problems. This study develops a new numerical model of the depth averaged horizontally 2-D SWEs, referred to as 2-D finite element/volume method (2-D FEVM) model. The continuity equation is solved with the conforming, standard Galerkin finite element method scheme and the momentum equations with an upwind, cell-centered finite volume method scheme, utilizing the water surface elevation and the line discharges as unknowns aligned in a staggered manner. The 2-D FEVM model relies on neither Riemann solvers nor high-resolution algorithms in order to serve as a simple numerical model. Water at a rest state is exactly preserved in the model. A fully explicit temporal integration is achieved in the model using an efficient approximate matrix inversion method. A series of test problems, containing three benchmark problems and three experiments of transcritical flows, are carried out to assess accuracy and versatility of the model.

Key words: 2-D SWEs; Finite element/volume method; Approximate matrix inversion method; Fully explicit temporal integration. 


\section{Introduction}

Analysis of surface water flows is of central importance in understanding and predicting a wide range of water engineering issues. Dynamics of surface water is reasonably well described using the shallow water equations (SWEs) with the hydrostatic pressure assumption. The SWEs consist of non-linear hyperbolic partial differential equations that describe conservation of mass and momentum in a surface water body as an incompressible fluid. Although the SWEs are simple compared with the three-dimensional non-hydrostatic models [1-3], they adequately describe various practically important flows such as river flows, dam break flash floods, and tidal flows in estuaries [4-6]. The SWEs have also been used in analyzing transport phenomena in shallow water bodies $[7,8]$.

Analytical solutions to the SWEs are available only for a limited number of cases [9-12]. The SWEs are therefore in general numerically solved in applications. Numerical models based on the cross-sectionally averaged 1-D and the depth averaged horizontally 2-D SWEs have extensively been applied to both scientific and engineering problems. Researches showed that the 1-D models are sufficient for simulating the flows traveling down narrow and steep channels [13-15]. 1-D models equipped with internal boundary conditions have been used in simulating the flows in open channel networks, such as urban and agricultural drainage systems, river networks, and multi-channel estuaries [16-18]. On the other hand, 2-D models are necessary when the flow has inherently 2-D structures in the horizontal directions such that 1-D models cannot appropriately capture $[19,20]$.

Among various numerical models of the SWEs, those utilizing the finite volume method (FVM) have been accepted as the most effective because of their inherent conservation property and high ability to handle flows in complex geometries. Most of the FVM models are based on Riemann solvers, which use approximate [21, 22] or exact solutions [23] to local Riemann problems in evaluation of numerical fluxes. Toro and Garcia-Navarro [24] provided an extensive review of the Riemann solvers. High-resolution algorithms such as slope limiter [25, 26] and adaptive remeshing [27, 28] also are widely used numerical techniques.

On the other hand, some researchers developed simple and sufficiently accurate numerical models of the SWEs utilizing neither Riemann solvers nor high-resolution algorithms. Van Reeuwijk [29] proposed a central FVM model of the 2-D SWEs for structured mesh that correctly conserves both mass, momentum, and energy, which has later been extended to be used with unstructured mesh [30]. Shi et al. [31] developed a projection type FVM model of the viscous 2-D SWEs having source terms. Finite element method (FEM) models and FVM models based on dual mesh (staggered mesh), such as the median mesh [32] and the Voronoi diagram [33], have practically been used because of their high abilities to suppress spurious oscillations and accuracy to conserve both mass and momentum $[34,35]$. Analogous numerical models have been applied to both the compressible and incompressible Navier-Stokes equations [36, 37], the Euler equations [30, 38], and the Boussinesq equations for dispersive wave dynamics [39]. Numerical models concurrently using both the FEM and FVM techniques, here referred to as FEVM (Finite Element/Volume Method) models, are among the most simple and effective ones to solve the SWEs [40, 41]. Compatibility between FEM schemes and cell-centered FVM schemes helps develop successful FEVM models that take 
advantages of both of the numerical schemes [42-44]. Sparse element formulation for the SWEs presented in Mewis [45], which uses linear and constant basis for the water depth and the velocities, respectively, can be regarded as an FEVM model. Wang and Liu [46] developed an FEVM model of the viscous 2-D SWEs. One of the authors showed that the 1-D FEVM models for the 1-D SWEs that apply the standard Galerkin FEM scheme and an upwind, cell-centered FVM scheme to the continuity equation and the momentum equation, respectively, exhibit satisfactory accuracy to simulate subcritical, supercritical, and transcritical flows in both idealized and engineering problems [47-49]. The FEVM models consistently deal with channel bends and junctions in terms of a corrected momentum flux method. The use of a staggered alignment for the unknowns, which are the nodal water surface elevation and the elemental discharge, is essential in the 1-D FEVM models to compute steady and transient solutions that the conventional FEM models fail [50, 51]. FVM counterparts of the 1-D FEVM models have also been developed by the authors [52, 53]. Artificial dissipative terms and shock capturing terms [54-56] are not utilized in the 1-D FEVM models. The FEM scheme used in the models are conforming in terms of that their test functions are globally continuous, which is not the case for some of the Petrov-Galerkin FEM schemes [57, 58]. The 1-D FEVM models exactly preserve water at a rest state, and do not utilize any water surface reconstruction algorithms [59-61]. The 1-D FEVM models are sufficiently accurate and robust for a wide range of problems; however, clearly the models cannot be applied to the flows having horizontally 2-D structures.

This study develops a new FEVM model of the 2-D SWEs, referred to as 2-D FEVM model, for subcritical, supercritical, and transcritical flows with horizontally 2-D structures such that 1-D models cannot appropriately capture. The 2-D FEVM model is a 2-D counterpart of the 1-D FEVM models [47-49]. Spatial discretization of the model is based on triangular mesh. As with the 1-D FEVM model, the continuity equation is solved using the standard Galerkin FEM scheme with linear basis and the momentum equations using an upwind, cell-centered finite FVM scheme, so that discrete conservation of mass and momentum are achieved without complex interpolation algorithms. Since the 2-D FEVM model uses a non-lumped discretization for the temporal term as in typical FEM models, application of a linear system solver is required at each time step to carry out the temporal integration, even if an explicit method is utilized. This problem can be avoided by the use of a mass-lumping for the temporal term analogous to some of the vertex-centered FVM models [62, 63]. Although the mass-lumping serves as a simple and efficient computational strategy, researches pointed out its disadvantages that should not be overlooked. Application of the mass-lumping results in the loss of phase accuracy [64, 65] and adds significant artificial dissipation [66]. Fang and Sheu [67] proposed an efficient and accurate iterative temporal integration method for an FEM model of the 2-D SWEs, which is based on a combined use of both lumped and non-lumped mass matrices. Guermond and Pasuquetti [68] developed an approximated matrix inversion method for the non-lumped discretization of hyperbolic partial differential equations, which does not rely on any linear system solvers and thus does not spoil advantages of the explicit methods. Application of such an effective algorithm is necessary in practical analysis so that both accuracy and efficiency of a model are not degraded. Temporal integration of the 2-D FEVM model is therefore carried out using the matrix inversion algorithm [68]. Accuracy of the model is verified with a series of test problems that contain three 
benchmark problems and three experiments of transcritical flows. The last one of the experimental flows in particular serves as a severe 2-D test case that involves conversion and diversion of the flows, a hydraulic jump, oblique shocks, and a hydraulic bore propagating upstream.

The remainder of this paper is organized as follows. A concise introduction is given for the 2-D SWEs in Section 2. The 2-D FEVM model is presented in Section 3. In Section 4, the 2-D FEVM model is verified with the test problems. Section 5 provides conclusions of this study.

\section{2-D SWES}

The 2-D SWEs represent conservation of mass and momentum in a hydrostatic surface water body [69]. The 2-D SWEs used in this study are in a conservative form, which are appropriate for simulating not only gradually varied flows but also rapidly varied flows involving transitions. The 2-D SWEs on the Cartesian $x-y-z$ coordinates consist of the continuity equation

and the momentum equations

$$
\frac{\partial \eta}{\partial t}+\frac{\partial p}{\partial x}+\frac{\partial q}{\partial y}=0
$$

$$
\frac{\partial p}{\partial t}+\frac{\partial}{\partial x}\left(\frac{p^{2}}{h}\right)+\frac{\partial}{\partial y}\left(\frac{p q}{h}\right)+g h\left(\frac{\partial \eta}{\partial x}+f_{x}\right)=0
$$

and

$$
\frac{\partial q}{\partial t}+\frac{\partial}{\partial x}\left(\frac{p q}{h}\right)+\frac{\partial}{\partial y}\left(\frac{q^{2}}{h}\right)+g h\left(\frac{\partial \eta}{\partial y}+f_{y}\right)=0
$$

where $t$ is the time, $\eta$ is the water surface elevation, $h(=\eta-z)$ is the water depth, $g$ is the acceleration due to gravity, $p$ and $q$ are the line discharges in the $x$ and $y$ directions, respectively. $f_{x}$ and $f_{y}$ are the friction slopes in the $x$ and $y$ directions, which are given by the Manning's formulae

$$
f_{x}=\frac{n^{2}}{h^{10 / 3}} p \sqrt{p^{2}+q^{2}}
$$

and

$$
f_{y}=\frac{n^{2}}{h^{10 / 3}} q \sqrt{p^{2}+q^{2}},
$$

respectively, where $n$ is the Manning's coefficient which is assumed constant in this study.

\section{Finite element/volume method (FEVM) model}

\subsection{Computational mesh}

The proposed 2-D FEVM model applies the standard Galerkin FEM scheme with linear basis to the continuity equation (1) and an upwind, cell-centered FVM scheme to the momentum equations (2) and (3). The model uses the water surface elevation $\eta$ 
and the line discharges $p$ and $q$ as unknowns. The horizontally 2-D domain $\Omega$ is firstly divided into a triangular mesh consisting of non-overlapping regular triangular elements [70]. The elements and the nodes are indexed with the natural numbers. The total numbers of elements and nodes are denoted by $\mathrm{N}_{\mathrm{e}}$ and $\mathrm{N}_{\mathrm{n}}$, respectively. The $i$ th node is denoted by $\mathrm{P}_{i}$. The $k$ th element is denoted by $\Omega_{k}$. The area of $\Omega_{k}$ is represented by $A_{k}$. The three edges of $\Omega_{k}$ are denoted counterclockwise by $e(k, 1)$ th edge, $e(k, 2)$ th edge, and $e(k, 3)$ th edge. Length of $e(k, m)$ is denoted by $l_{k, m}$. The two nodes that bound the edge $e(k, m)$ are denoted as $\varepsilon(k, m, 1)$ th node and $\varepsilon(k, m, 2)$ th node in an counterclockwise manner. The element that shares the edge $e(k, m)$ with $\Omega_{k}$ is denoted by the $\imath(k, m)$ th element, namely $\Omega_{l(k, m)}$. The number of elements that share $\mathrm{P}_{i}$ is denoted by $v(i)$. The $j$ th element shearing $\mathrm{P}_{i}$ is denoted as $\Omega_{\kappa(i, j)}$. The element $\Omega_{\kappa(i, j)}$ has three nodes; one of them is the $i$ th node, and the others are referred to as the $\mu(i, j, 1)$ th node and the $\mu(i, j, 2)$ th node in a counterclockwise manner. Length of the edge bounded by $\mathrm{P}_{\mu(i, j, 1)}$ and $\mathrm{P}_{\mu(i, j, 2)}$ is denoted by $L_{i, j}$. Figures 1(a) and 1(b) show schematic diagrams of the triangular mesh. The water surface elevation $\eta$ is attributed to the nodes whereas the line discharges $p$ and $q$ to the elements. The nodal $\eta$ at $\mathrm{P}_{i}$ is denoted by $\eta_{i}$. Distribution of the discretized $\eta$ within an element is assumed to linearly interpolate the nodal values. The water depth $h$ and the bed topography $z$ are distributed to the nodes in the same way as the water surface elevation $\eta$. The elemental $p$ and $q$ in $\Omega_{k}$ are denoted as $p_{k}$ and $q_{k}$, respectively. Application of the 2-D FEVM model to the problems with wet and dry interfaces is not the subject of this study, and will be focused on in future works. The water depth $h$ is therefore assumed to be positive in this study.

(a)

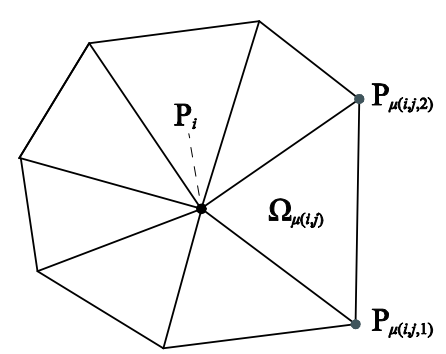

(b)

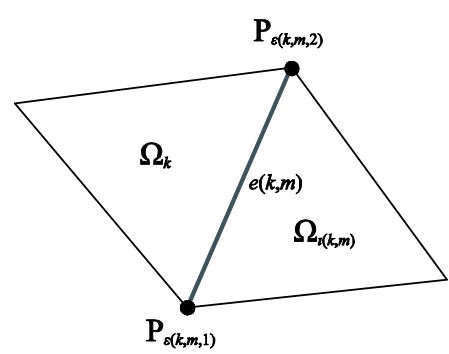

Figure 1 Schematic diagrams of triangular mesh. 


\subsection{Spatial discretization}

\subsubsection{Continuity equation}

The continuity equation (1) can be represented as the weak form

$$
\int_{\omega} w \frac{\partial \eta}{\partial t} \mathrm{~d} x \mathrm{~d} y-\int_{\omega}\left(\frac{\partial w}{\partial x} p+\frac{\partial w}{\partial y} q\right) \mathrm{d} x \mathrm{~d} y=Q^{*}
$$

for a test function $w$ with its compact support $\omega$ in $\Omega$. The right hand side of (6), namely $Q^{*}$, represents boundary integral terms. The standard Galerkin FEM scheme with the continuous, linear basis on the triangular mesh is used to spatially discretize (6). The weight function associated with the node $\mathrm{P}_{i}$ is denoted by $w_{i}$. The support of $w_{i}$, denoted by $\omega_{i}$, is given by the closed set of the elements shearing the node $\mathrm{P}_{i}$ as

$$
\omega_{i}=\overline{\bigcup_{j=1}^{v(i)} \Omega_{\kappa(i, j)}} .
$$

Substituting $w_{i}$ into (6) yields

$$
\int_{\omega_{i}} w_{i} \frac{\partial \eta}{\partial t} \mathrm{~d} x \mathrm{~d} y-\int_{\omega_{i}}\left(\frac{\partial w_{i}}{\partial x} p+\frac{\partial w_{i}}{\partial y} q\right) \mathrm{d} x \mathrm{~d} y=Q^{*}
$$

The first term of the left hand side of (8), namely the temporal term, is calculated as

$$
\begin{aligned}
\int_{\omega_{i}} w_{i} \frac{\partial \eta}{\partial t} \mathrm{~d} x \mathrm{~d} y & =\sum_{j=1}^{v(i)} \int_{\Omega_{\kappa(i, j)}} w_{i} \frac{\partial \eta}{\partial t} \mathrm{~d} x \mathrm{~d} y \\
& =\sum_{j=1}^{v(i)} A_{\kappa(i, j)}\left(\frac{1}{6} \frac{\mathrm{d} \eta_{i}}{\mathrm{~d} t}+\frac{1}{12} \frac{\mathrm{d} \eta_{\mu(i, j, 1)}}{\mathrm{d} t}+\frac{1}{12} \frac{\mathrm{d} \eta_{\mu(i, j, 2)}}{\mathrm{d} t}\right) .
\end{aligned}
$$

The second term of the left hand side of (8) is calculated as

$$
-\int_{\omega_{i}}\left(\frac{\partial w_{i}}{\partial x} p+\frac{\partial w_{i}}{\partial y} q\right) \mathrm{d} x \mathrm{~d} y=\sum_{j=1}^{v(i)} \frac{L_{i, j}}{2}\left(p_{\Omega_{\kappa(i, j)}} n_{i, j, x}+q_{\Omega_{\kappa(i, j)}} n_{i, j, y}\right)
$$

where $\left(n_{i, j, x}, n_{i, j, y}\right)$ is the outward unit normal vector on the edge bounded by the nodes $\mathrm{P}_{\mu(i, j, 1)}$ and $\mathrm{P}_{\mu(i, j, 2)}$. Consequently, (8) results in $\sum_{j=1}^{v(i)} A_{\kappa(i, j)}\left(\frac{1}{6} \frac{\mathrm{d} \eta_{i}}{\mathrm{~d} t}+\frac{1}{12} \frac{\mathrm{d} \eta_{\mu(i, j, 1)}}{\mathrm{d} t}+\frac{1}{12} \frac{\mathrm{d} \eta_{\mu(i, j, 2)}}{\mathrm{d} t}\right)+\sum_{j=1}^{v(i)} \frac{L_{i, j}}{2}\left(p_{\Omega_{\kappa(i, j)}} n_{i, j, x}+q_{\Omega_{\kappa(i, j)}} n_{i, j, y}\right)=Q^{*}$

The proposed spatial discretization for the continuity equation ensures conservation of mass because the second term of the left hand side of (11) is in a flux form analogous to some of the vertex-centered FVM schemes [63].

\subsubsection{Momentum equations}

An upwind, cell-centered FVM scheme on the triangular mesh is used to spatially discretize the momentum equations (2) and (3). Application of the FVM scheme to (2) and (3) on the element $\Omega_{k}$ as a control volume leads to 


$$
A_{k} \frac{\mathrm{d} p_{k}}{\mathrm{~d} t}+\sum_{m=1}^{3} l_{k, m}\left(p V_{n}\right)_{e(k, m)}+\int_{A_{k}} g h\left(\frac{\partial \eta}{\partial x}+f_{x}\right) \mathrm{d} x \mathrm{~d} y=0
$$

and

$$
A_{k} \frac{\mathrm{d} q_{k}}{\mathrm{~d} t}+\sum_{m=1}^{3} l_{k, m}\left(q V_{n}\right)_{e(k, m)}+\int_{A_{k}} g h\left(\frac{\partial \eta}{\partial y}+f_{y}\right) \mathrm{d} x \mathrm{~d} y=0,
$$

respectively, where $V_{n}$ is the velocity defined on the boundary of $\Omega_{k}$. The momentum fluxes $\left(p V_{n}\right)_{e(k, m)}$ and $\left(q V_{n}\right)_{e(k, m)}$ on the edge $e(k, m)$ are determined from an upwind evaluation method utilizing local Froude number as a weight. Let $\phi$ denote $p$ or $q$. The momentum flux evaluation method presented here is a 2-D counterpart of that used in the 1-D FEVM model [49]. The method is heuristic, but its accuracy for 1-D problems was extensively verified through a number of benchmark problems. First, the velocity on the edge $e(k, m)$ of $\Omega_{k}$ is calculated as

$$
V_{e(k, m)}=\frac{1}{\left(1-\theta_{k, m}\right) \bar{h}_{e(k, m)}+\theta_{k, m} h_{k, m, \mathrm{up}}}\left(p_{k} \hat{n}_{k, m, x}+q_{k} \hat{n}_{k, m, y}\right)
$$

with the upwinding index

$$
\theta_{k, m}=\max \left(1-\frac{1}{F r_{k, m}^{2}}, 0\right)
$$

and the square of local Froude number

$$
F r_{k, m}{ }^{2}=\frac{p_{k}{ }^{2}+q_{k}{ }^{2}}{g\left(\bar{h}_{e(k, m)}\right)^{3}}
$$

where $\left(\hat{n}_{k, m, x}, \hat{n}_{k, m, y}\right)$ is the outward unit normal vector on $e(k, m), \bar{h}_{e(k, m)}$ is the averaged water depth on $e(k, m)$, which is calculated as

$$
\bar{h}_{e(k, m)}=\frac{1}{2}\left(h_{\varepsilon(k, m, 1)}+h_{\varepsilon(k, m, 2)}\right) \text {. }
$$

The upwinding index $\theta_{k, m}$ in (15) is determined so that the momentum flux is evaluated using the information of the upstream when the flow is supercritical depending on the magnitude of the local Froude number $F r_{k, m}$. The upwind water depth $h_{k, m \text {,up }}$ is determined as follows. Let $\lambda_{k, m}$ be the straight line parallel to the vector $\left(p_{k}, q_{k}\right)$ and passing the middle point of the edge $e(k, m)$. If the vector $\left(p_{k}, q_{k}\right)$ is directing outward of $e(k, m)$ then the line $\lambda_{k, m}$ intersects with the boundary of $\Omega_{k}$ at another point and $h_{k, m \text {,up }}$ is determined as the water depth at that point. Otherwise, $h_{k, m, \text { up }}$ is set equal to $\bar{h}_{e(k, m)}$. The elemental momentum flux $\left(\phi V_{n}\right)_{e(k, m)}$ on $e(k, m)$ of the triangular cell $\Omega_{k}$ is determined as

$$
\left(\phi V_{n}\right)_{e(k, m)}=\phi_{k} V_{e(k, m)} .
$$

The momentum flux on $e(k, m)$ is finally determined by extending up the elemental 
fluxes in $\Omega_{k}$ and $\Omega_{\imath(k, m)}$ as

$$
\left(\phi V_{n}\right)_{e(k, m)}=H\left(V_{e(k, m)}\right) \phi_{k} V_{e(k, m)}-H\left(-V_{e\left(l(k, m), m^{\prime}\right)}\right) \phi_{l(k, m)} V_{e\left(l(k, m), m^{\prime}\right)}
$$

where $H$ is the Heaviside's step function and the integer $m^{\prime}$ is chosen so that the edge $e(k, m)$ coincides with the edge $e\left(l(k, m), m^{\prime}\right)$. Momentum fluxes are directly specified if necessary when the edge $e(k, m)$ is a part of the boundary of the domain $\Omega$.

The gravitational source terms in (12) and (13) are evaluated on the basis of the linearity of $\eta, h$, and $z$ in $\Omega_{k}$ as

$$
\int_{A_{k}} g h \frac{\partial \eta}{\partial x} \mathrm{~d} x \mathrm{~d} y=\left.A_{k} g \bar{h}_{k} \frac{\partial \eta}{\partial x}\right|_{\Omega_{k}}
$$

and

$$
\int_{A_{k}} g h \frac{\partial \eta}{\partial y} \mathrm{~d} x \mathrm{~d} y=\left.A_{k} g \bar{h}_{k} \frac{\partial \eta}{\partial y}\right|_{\Omega_{k}}
$$

where $\bar{h}_{k}$ is the water depth at the center of gravity of $\Omega_{k}$. The partial differences $\left.\frac{\partial \eta}{\partial x}\right|_{\Omega_{k}}$ and $\left.\frac{\partial \eta}{\partial y}\right|_{\Omega_{k}}$ in (20) and (21) are spatially constant in $\Omega_{k}$, which are accordingly calculated utilizing the fact that the water surface elevation $\eta$ is linearly interpolated in each element. Evaluation of the friction slopes $f_{x}$ and $f_{y}$ is carried out using the elemental values $\bar{h}_{k}, p_{k}$ and $q_{k}$. The 2-D FEVM model exactly preserves water at a rest state without any special treatments of the source terms because it discretizes the gravitational source terms directly utilizing the water surface elevation $\eta$ as shown in (20) and (21).

\subsection{Temporal integration}

The 2-D FEVM model uses an explicit method in its temporal integration. The second-order Runge-Kutta method [71] is applied to the temporal integration incorporating boundary conditions if necessary. A higher order method, such as the fourth-order Runge-Kutta method, is also implementable in the temporal integration; however, preliminary computation showed that the application of the second-order Runge-Kutta method is satisfactory for the problems presented in the next section. The 2-D FEVM model utilizes the efficient approximated matrix inversion method proposed in Guermond and Pasuquetti [68] so that a fully explicit temporal integration is achieved. Now the inverse of the mass matrix $M=\left[M_{i, i^{\prime}}\right]$ for the spatially discretized continuity equation (11), which is given by an $\mathrm{N}_{\mathrm{n}}$-dimensional positive definite matrix, is approximated as

$$
M^{-1} \approx\left(I+\sum_{n=1}^{d} B^{n}\right) N^{-1}
$$

where $I$ is the $\mathrm{N}_{\mathrm{n}}$-dimensional identity matrix, $d \geq 1$ is a fixed integer, $B$ is the 
$\mathrm{N}_{\mathrm{n}}$-dimensional square matrix whose spectral radius is always smaller than 1 , which is defined as

$$
B=N^{-1}(N-M),
$$

and $N=\operatorname{diag}\left\{N_{1}, \ldots, N_{\mathrm{N}_{\mathrm{n}}}\right\}$ is the $\mathrm{N}_{\mathrm{n}}$-dimensional lumped mass matrix whose $i$ th entry is given by

$$
N_{i}=\sum_{i^{\prime}=1}^{\mathrm{N}_{\mathrm{n}}} M_{i, i^{\prime}} .
$$

In this study, the integer $d$ in (22) is fixed to 2 as Guermond and Pasuquetti [68] recommended. It has been confirmed that increasing $d$ does not significantly alter the numerical solutions presented below.

\section{Numerical tests}

The 2-D FEVM model is validated through a series of test problems containing three benchmark problems and three hydraulic experiments; these are steady flows over a bump [72], a partial dam break problem [73], an oblique hydraulic jump [74], steady transcritical flows in a sharply bending channel [48], and hydraulic bores in a rectangular flume with and without an obstacle [49]. Computational meshes for the test problems are created using a free unstructured mesh generator Voro Ver 3.17 (available at http://www32.ocn.ne.jp/ yss/voro.html.).

\subsection{Steady flows over a bump}

Steady flows over a bump are standard test problems to check accuracy of a numerical model to discretize the gravitational source terms of the momentum equations with a non-flat bed [72]. A $20 \mathrm{~m}$ long frictionless rectangular channel with the width of $0.6 \mathrm{~m}$ is considered as the domain $\Omega=(0 \mathrm{~m}, 20 \mathrm{~m}) \times(0.0 \mathrm{~m}, 0.6 \mathrm{~m})$. The bed topography of the channel is given by

$$
z=\max \left\{0,0.2-0.05(x-10)^{2}\right\} .
$$

Three different cases, which are BUMPs A through $\mathrm{C}$, are examined. Inflow line discharges for BUMPs A, B, and C are specified as $4.42 \mathrm{~m}^{2} / \mathrm{s}, 1.53 \mathrm{~m}^{2} / \mathrm{s}$, and $0.18 \mathrm{~m}^{2} / \mathrm{s}$, respectively. Analytical steady solutions under the different boundary conditions are obtained with the help of Bernoulli's principle and the relation of the sequent depth for a hydraulic jump. The analytical steady solutions to BUMPs A, B, and C are a subcritical flow with a depression on the bump, a transcritical flow without hydraulic jump, and a transcritical flow with a hydraulic jump at the position of $x=11.667 \mathrm{~m}$, respectively. These solutions are essentially 1-D along the channel and do not vary in the $y$ -direction, namely $q=0 \mathrm{~m}^{2} / \mathrm{s}$ in the entire domain. Initial guesses are fixed to $\eta=0.5$ $\mathrm{m}$ and $p=q=0 \mathrm{~m}^{2} / \mathrm{s}$ in the entire domain. Inflow momentum flux $F_{\text {in }}$ is prescribed on the upstream boundary of the channel as

$$
F_{\text {in }}=\frac{p_{\text {in }}^{2}}{h_{\text {in }}}
$$


where $p_{\text {in }}$ is the inflow line discharge in the $x$ direction and $h_{\text {in }}$ is the water depth at the middle point of the edge where the momentum flux is specified. The exact downstream water depths of $h=2.00 \mathrm{~m}$ and $h=0.33 \mathrm{~m}$ are directly specified in BUMPs $\mathrm{A}$ and $\mathrm{C}$ because their analytical solutions present subcritical outflows. On the other hand, in BUMP B, a free outflow boundary condition is specified on the downstream boundary because its analytical solution presents a supercritical outflow. A slip condition is specified on side walls of the channel. The domain $\Omega$ is discretized into a computational mesh with $\mathrm{N}_{\mathrm{e}}=3,992$ elements and $\mathrm{N}_{\mathrm{n}}=2,255$ nodes. The time increment is set as $0.0025 \mathrm{~s}$.

Figure 2 shows the analytical steady water surface profile and the scatter plots of the computed steady nodal water depths for each computational case. Steady numerical solutions are successfully obtained starting from the specified initial guesses incorporating the boundary conditions. Figure $\mathbf{3}$ shows an enlarged view of the computed water surface profile of BUMP C around the bump. Overall accuracy of the 2-D FEVM model is satisfactory, only minor errors in the water levels are observed for BUMPs A and B in particular. Computed transition from subcritical to supercritical in BUMP B is smooth similar to that of the analytical solution, which cannot be appropriately handled using some of the conventional models [75]. The constant water depth regions upstream and downstream of the bumps are correctly reproduced in each computational case. The computed solution for BUMP $\mathrm{C}$ is oscillatory around the hydraulic jump. The high-order polynomial interpolation functions and gradient reconstruction techniques for the source terms in the momentum equations in published models $[76,77]$ will suppress the numerical oscillations, but forfeiting the simplicity of the 2-D FEVM model. The order of the line discharge $q$ is smaller than $O\left(10^{-3}\right)$ $\mathrm{m}^{2} / \mathrm{s}$ in each computational case except for around the hydraulic jump in BUMP C where the maximum order of $q$ is $O\left(10^{-2}\right)$. However, the position of the hydraulic jump is accurately reproduced and the oscillations are observed only around the hydraulic jump not spreading out in the entire domain as in some of the conventional numerical models [78]. 


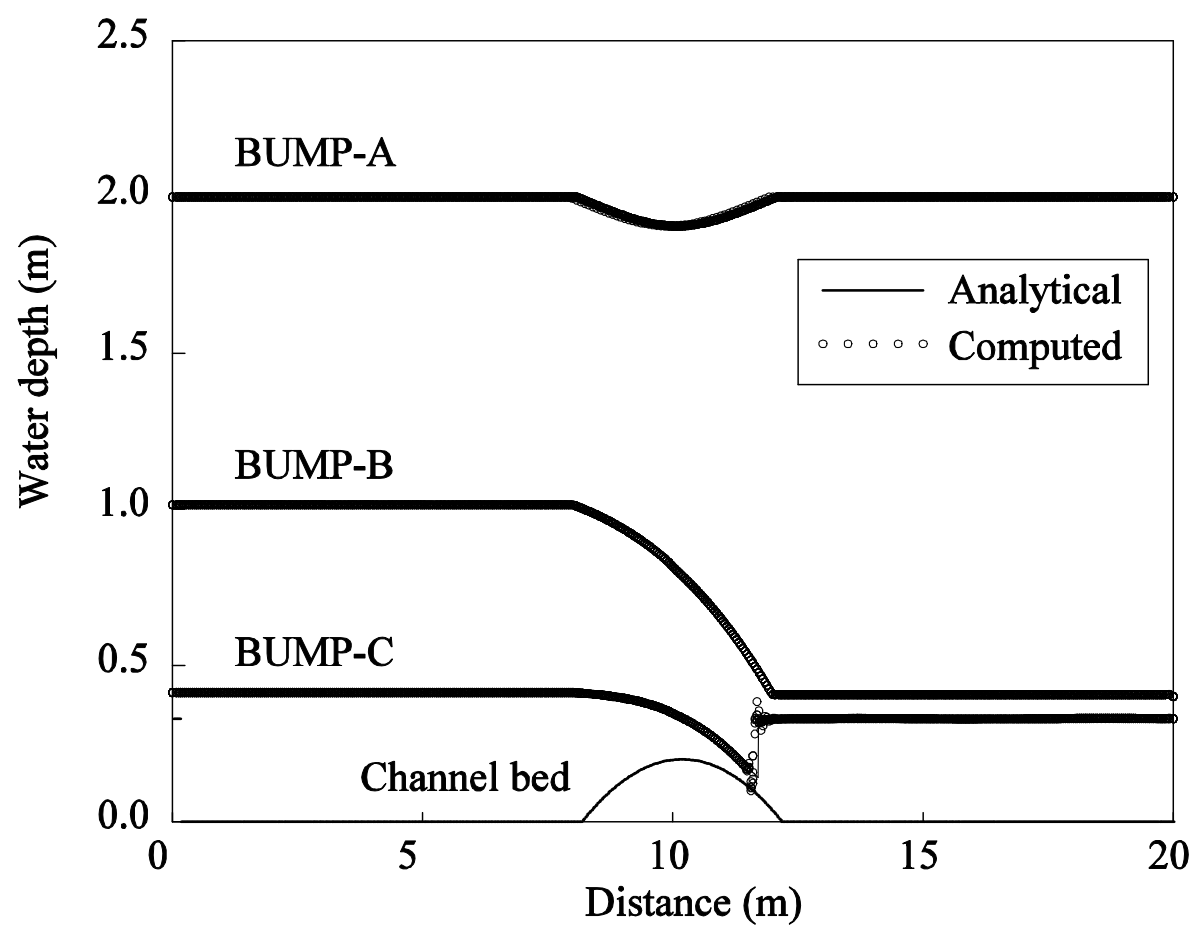

Figure 2 Computed water surface profiles for the steady flows over a bump.

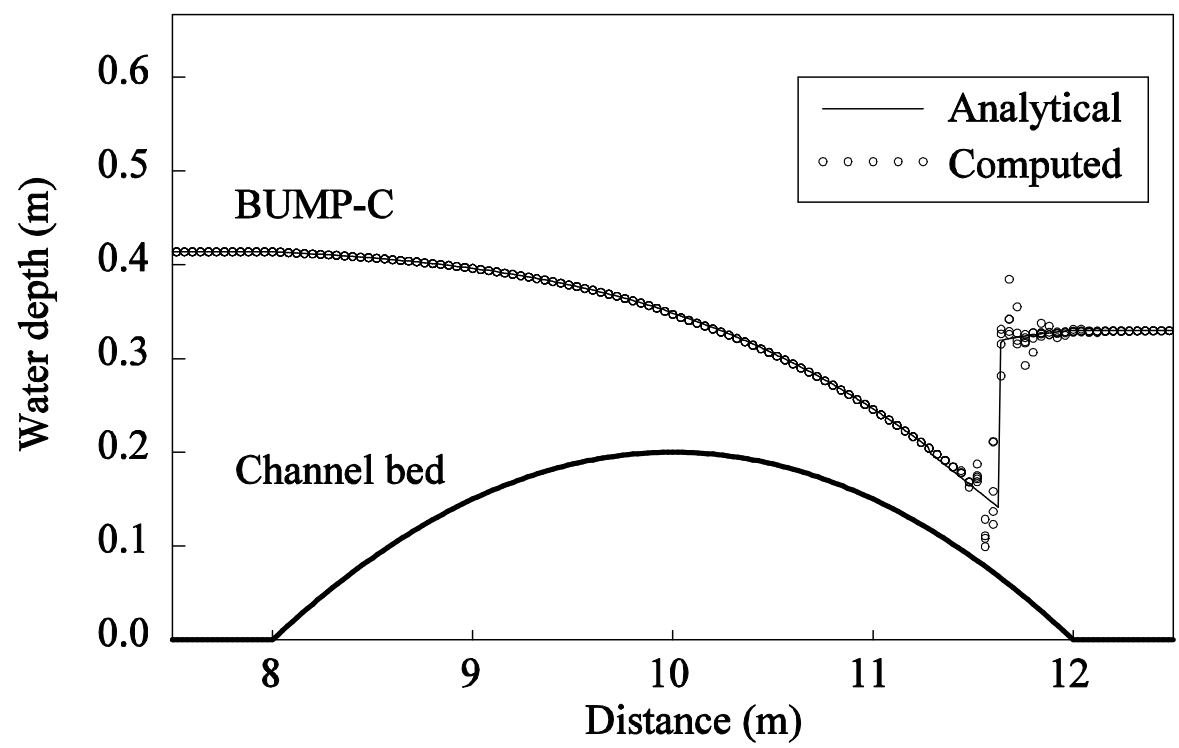

Figure 3 Enlarged view of the computed water surface profile for BUMP C.

4.2 Partial dam break problem

The 2-D FEVM model is verified with a partial dam break problem in a flat and frictionless basin. The model with the lumped mass matrix $N$, referred to as the 
lumped model, is also applied to the problem to see influence of the mass-lumping on dispersive and dissipative natures of numerical solutions. For this problem, the 2-D FEVM model is referred to as the original model to distinguish it from the lumped counterpart. Figure 4 shows the computational domain $\Omega$ for this test problem. Two regions of still water with the water depths of $10 \mathrm{~m}$ (upstream basin) and $5 \mathrm{~m}$ (downstream basin) are separated by an infinitesimally thin dam set along the line $x=100 \mathrm{~m}$, which is instantaneously and partially removed with the width of $75 \mathrm{~m}$ at the initial time $t=0 \mathrm{~s}$. The partial removal of the dam generates a bore progressing downstream and a depression wave moving upstream. This type of test problems has widely been used as important benchmarks to verify capability of numerical models to handle transient flows involving bores and depression waves [79-86]. The domain $\Omega$ is discretized into a computational mesh with $\mathrm{N}_{\mathrm{e}}=6,738$ elements and $\mathrm{N}_{\mathrm{n}}=3,523$ nodes. The time increment is set as $0.01 \mathrm{~s}$.

Figures 5 and $\mathbf{6}$ show the computed water surface profiles with the original model and the lumped model at the time $t=7.2 \mathrm{~s}$, respectively. The computed flow fields were entirely subcritical. Both the water surface profiles reasonably well capture the transient nature of the flow. As expected, the computational results are diffusive compared with those of the recent high-resolution models [85, 87]. Nevertheless, the results are less diffusive than those of the first order models $[88,89]$ and are comparable with some of the high-resolution models [90, 91].

The computational results with the two numerical models are qualitatively different; the lumped model preserves the monotonic surface water profiles of the depression wave upstream of the dam, while the original model gives slightly dispersive nature due to the use of the standard Galerkin FEM scheme. Similar results have been obtained for the 1-D models $[49,52]$. The computed front of the bore with the lumped model is slightly smoother than that of with the original model, indicating less dissipative nature of the approximated matrix inversion method used in this study. The computational results show validity of the proposed FEVM model against the transient flows with horizontally 2-D structures.

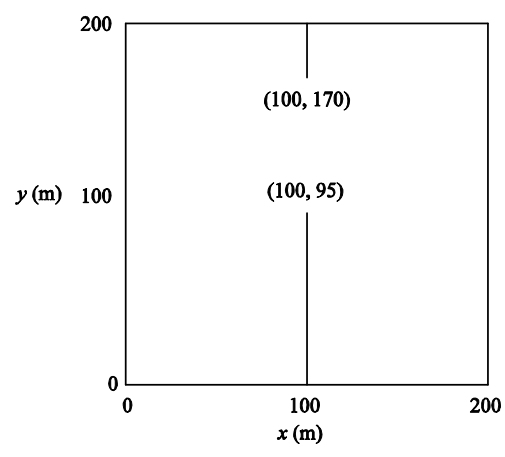

Figure 4 Computational domain for the partial dam break problem. 


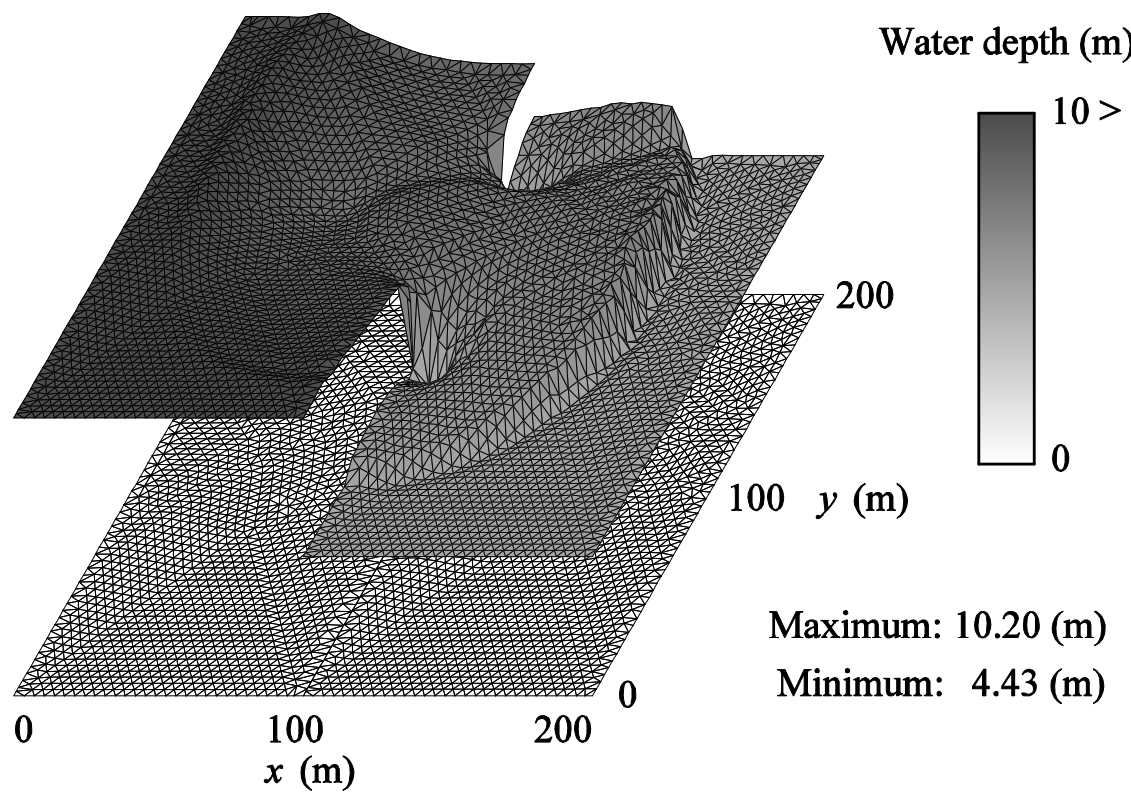

Figure 5 Computed water surface profile of the partial dam break problem using the original model.

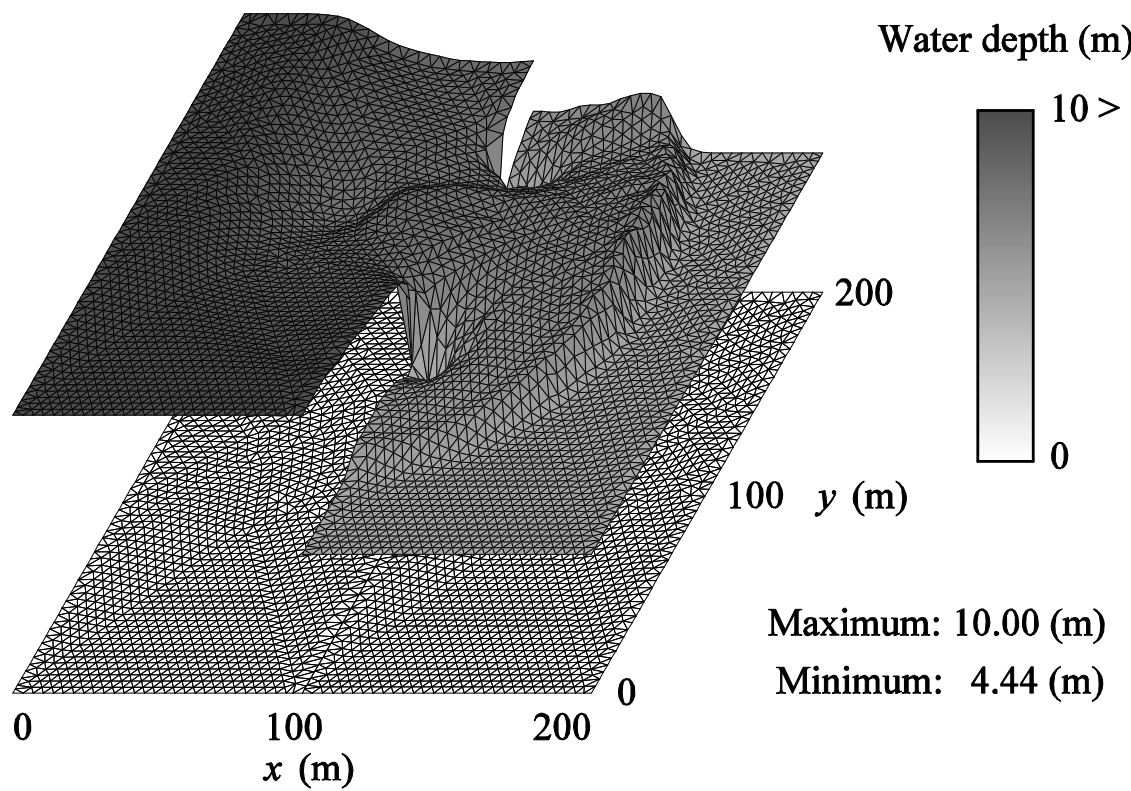

Figure 6 Computed water surface profile of the partial dam break problem using the lumped model.

\subsection{Oblique hydraulic jump}

An oblique hydraulic jump in a flat, frictionless converging channel is considered (Figure 7). An inflowing supercritical flow from the upstream boundary hits the convergent wall generating an oblique, supercritical hydraulic jump. Steady transition from supercritical to supercritical via a hydraulic jump cannot be reproduced by the 1-D SWEs. The angle between the converging wall and the inflow direction is set as 8.95 deg. A supercritical inflow with the water depth of $1.0 \mathrm{~m}$ and the velocity of $8.57 \mathrm{~m} / \mathrm{s}$ 
are imposed on the upstream boundary in terms of the line discharge and the momentum flux. A free outflow condition is specified on the downstream boundary. Other boundaries are treated as slip walls. Initial guesses are set as $\eta=h=1 \mathrm{~m}, p=8.57$ $\mathrm{m}^{2} / \mathrm{s}$, and $q=0 \mathrm{~m}^{2} / \mathrm{s}$, which amount to the Froude number of 2.74 . This test problem is a common benchmark to verify capability of numerical models to handle steady supercritical flows involving shocks. Analytical solution to this test problem is available in the literature [74]. According to Alcrudo and Garcia-Navarro [92], the analytical water depth and the velocity downstream of the jump are $1.5 \mathrm{~m}$ and $7.9556 \mathrm{~m} / \mathrm{s}$, respectively. The domain is discretized into a computational mesh with $\mathrm{N}_{\mathrm{e}}=4,163$ elements and $\mathrm{N}_{\mathrm{n}}=2,174$ nodes. The time increment is set as $0.0025 \mathrm{~s}$.

Figure 8 shows the steady water surface profile computed with the 2-D FEVM model. The computed water surface profile is oscillatory due to the absence of the limiting procedure in the model to preserve solution monotonicity; nevertheless, the computed position of the front of the oblique hydraulic jump agrees quite well with those of the analytical and the published results [73, 93, 94]. Oscillatory numerical solutions have also been obtained using the Godunov type model [27] and the Riemann solver with the superbee limiter [95]. The largest overshoot occurs at the downstream end of the front of the hydraulic jump common to the conventional FVM models [96]. The computed flow downstream of the jump was in good agreement with the published ones, within the relative errors almost less than $5 \%$.

Another numerical simulation using a finer computational mesh under the same initial and boundary conditions with $\mathrm{N}_{\mathrm{e}}=9,449$ elements and $\mathrm{N}_{\mathrm{n}}=4,862$ nodes is also carried out in order to see convergence of the numerical solution. Figure 9 shows the computed steady water surface profile with the fine mesh. Although the overshoot and undershoot of the water surface profile are not completely removed, the oblique hydraulic jump is more sharply captured than in the previous computational results. According to the computational results for this test problem, it is concluded that the 2-D FEVM model adequately handles steady supercritical flows with shocks despite its simplicity.

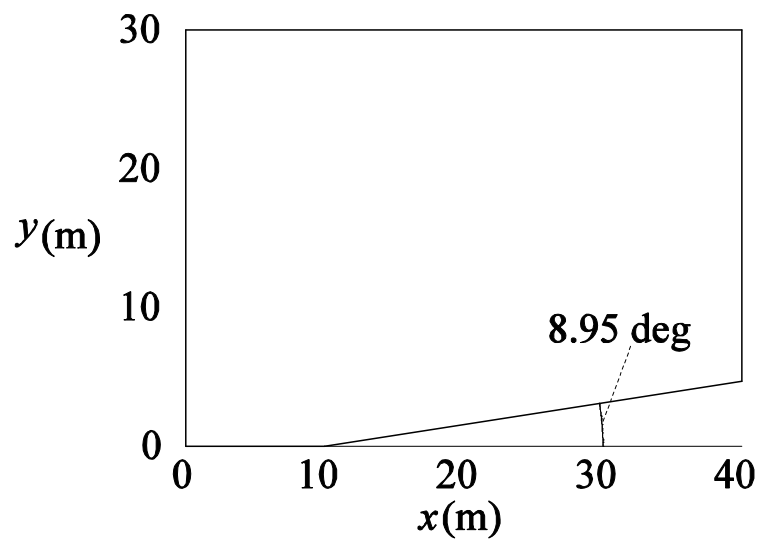

Figure 7 Computational domain for the oblique hydraulic jump. 


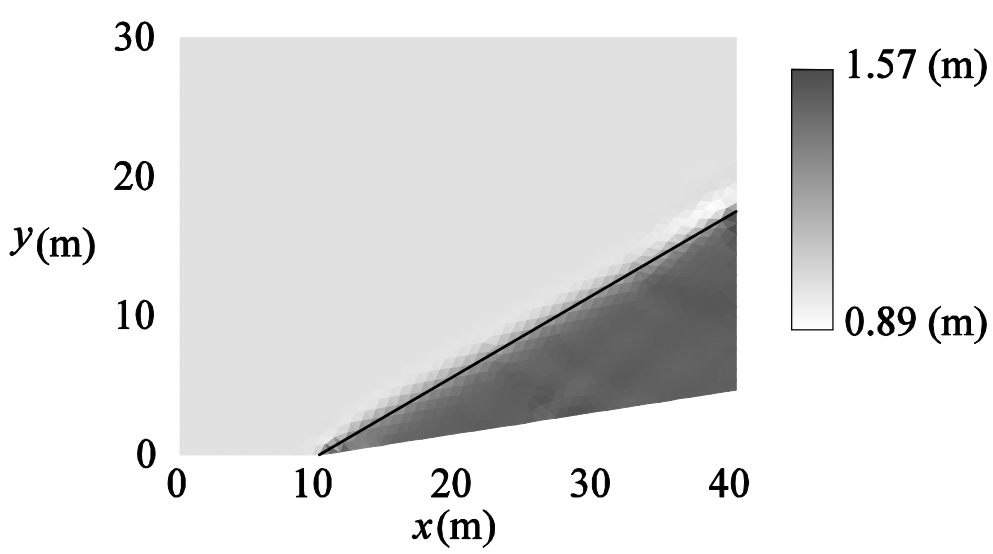

Figure 8 Computed water surface profile of the oblique hydraulic jump.

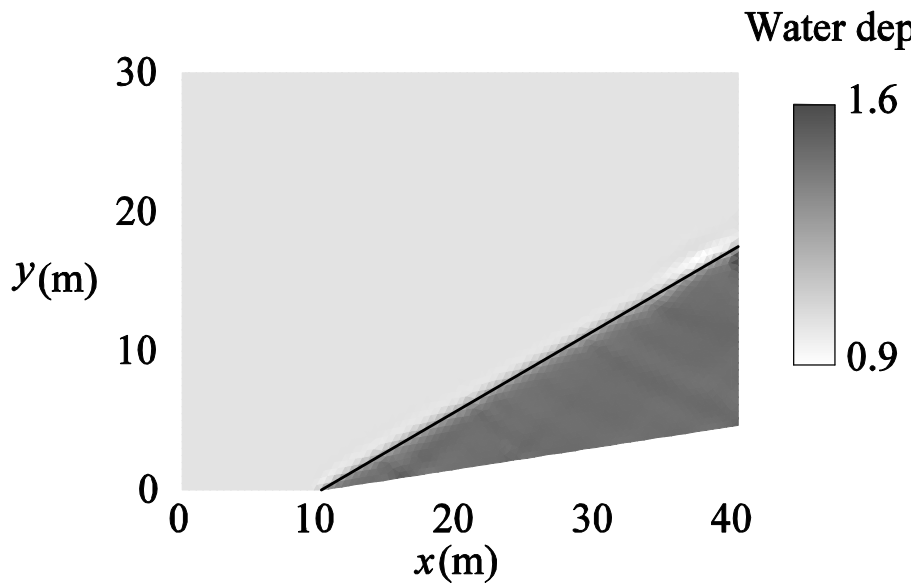

Figure 9 Computed water surface profile of the oblique hydraulic jump with the refined mesh.

\subsection{Steady transcritical flows in a bending channel}

Steady transcritical flows in a sharply bending channel are considered in order to see applicability of the 2-D FEVM model to experimental steady transcritical flows with shocks. Ishida et al. [48] carried out a series of laboratory experiments to validate a 1-D FEVM model against transcritical flows involving bends. Concrete blocks are configured in a rectangular flume to create a channel having two sharp bends. Figure 10 shows a schematic sketch of the channel serving as the domain $\Omega$. Bricks were arranged at the upstream end of the channel to generate a control section for flow regulation. Width and bed slope of the channel were $0.21 \mathrm{~m}$ and 0.01 , respectively. Downstream end of the channel was a free outfall. Three hydraulic experiments, which are referred to as Cases A through $\mathrm{C}$, were carried out in the channel. In each case, water was continuously provided from the pump. Water discharges continuously supplied by the pump were $3.70 \mathrm{~L} / \mathrm{s}, 4.55 \mathrm{~L} / \mathrm{s}, 5.25 \mathrm{~L} / \mathrm{s}$ in Cases A, B, and C, respectively. The water flowing down the channel hits the first bend generating a hydraulic jump in its upstream reach. Valiani and Caleffi [97] systematically investigated relevance of the 2-D SWEs for transcritical flows involving hydraulic jumps in bending channels. However, the front positions of the hydraulic jumps were not verified. Front position of the hydraulic 
jump in upstream of the first bend was therefore measured in each case. The hydraulic jumps were wavy and fluctuating, which are considered due to the slight instability of the discharges from the pump. The Manning's coefficients for the flume and the concrete block were estimated as $0.010 \mathrm{~s} / \mathrm{m}^{1 / 3}$ and $0.012 \mathrm{~s} / \mathrm{m}^{1 / 3}$, respectively [48]. However, the value for the channel was not identified. The Manning's coefficient for the channel should be chosen appropriately because it is considered to critically affect the front positions of the hydraulic jumps.

The domain $\Omega$ is discretized into a computational mesh with $\mathrm{N}_{\mathrm{e}}=4,299$ elements and $\mathrm{N}_{\mathrm{n}}=2,403$ nodes. Water discharge and the corresponding uniform water depth are specified on the upstream end and a free outflow condition is specified on the downstream end in each case. Side walls of the channel are treated as slip walls. The time increment is fixed to $0.005 \mathrm{~s}$. Numerical simulation in each case is carried out utilizing the water at a rest state with the water depth of $0.03 \mathrm{~m}$ as the initial guess, and is continued for sufficiently long time steps so that the flow reaches a steady state. On the basis of a try and error approach, the Manning's coefficient $n$ for the channel is estimated as $0.0101 \mathrm{~s} / \mathrm{m}^{1 / 3}$, which is determined so that the computed positions of the hydraulic jumps agree well with the observed ones. The front positions of the hydraulic jumps vary sensitively with $n$ in all the computational cases, modifying its value to $0.0001 \mathrm{~s} / \mathrm{m}^{1 / 3}$ shifts the position for several centimeters in some cases. The estimated value of the Manning's coefficient is considered acceptable because it is an intermediate value of that of the flume and the concrete blocks.

Figure 11 shows comparisons of the observed and computed front positions of the hydraulic jump for each case. The computed front positions with the 1-D FEVM model [48] are also shown in the figure. Observed front position of the hydraulic jump in each case, which was fluctuating, is plotted as a box to represent its fluctuation range. Both the 1-D and proposed 2-D models accurately capture the positions of the hydraulic jumps in all cases. The computed flow downstream of the hydraulic jump in each case presents 2-D structures involving a very shallow water region at the corner of the second bend such that 1-D models cannot capture. Although measured data to quantitatively validate the computed flows in the entire domain are not available, the results are qualitatively consistent with the observations [48]. The computational results show that the proposed model adequately simulates the transcritical flows involving shocks when the value of the Manning's coefficient is chosen appropriately.

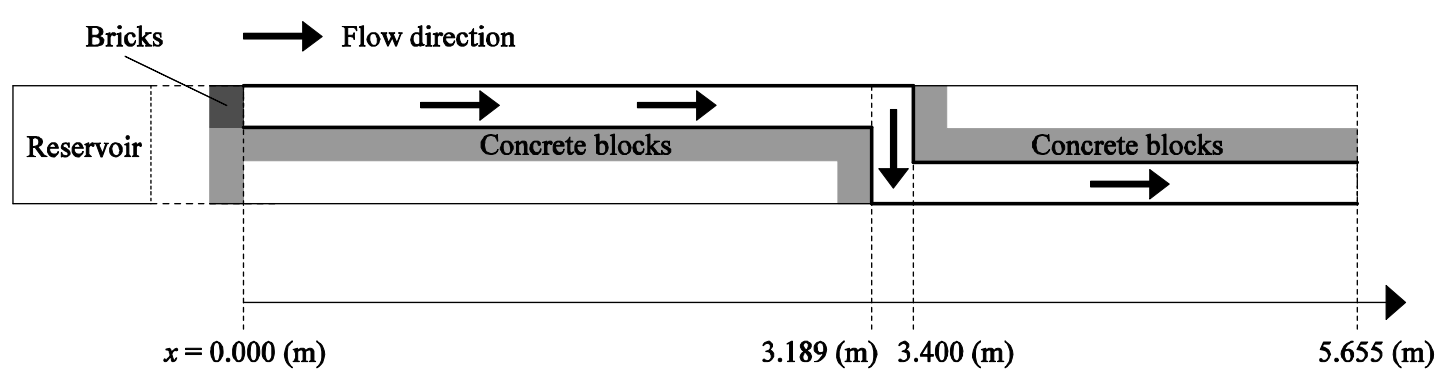

Figure 10 Schematic sketch of the bending channel. 


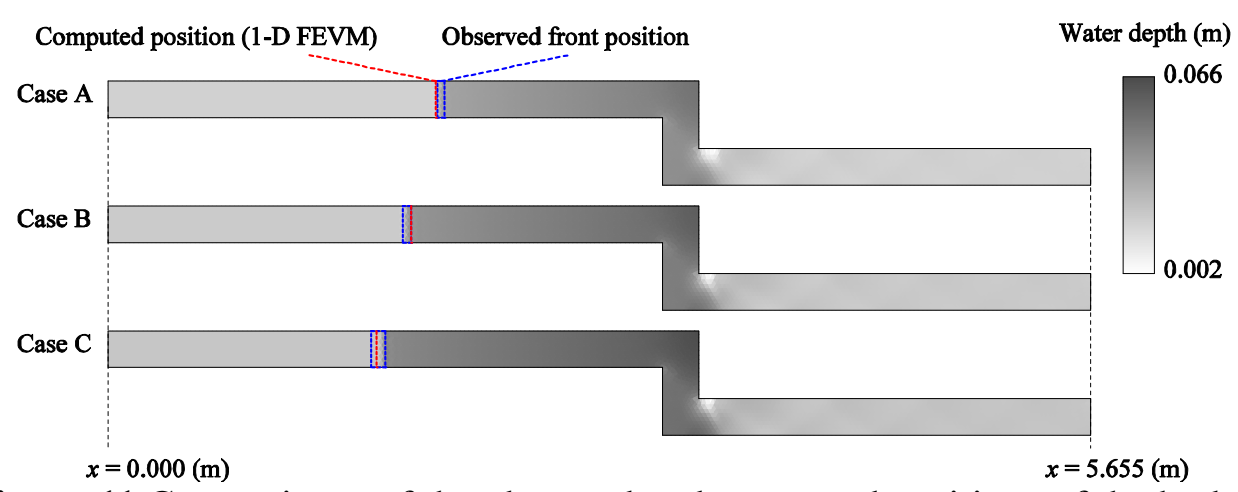

Figure 11 Comparisons of the observed and computed positions of the hydraulic jumps for each case.

\subsection{Hydraulic bore in a rectangular flume}

The 2-D FEVM model is applied to a hydraulic bore running up a supercritical uniform flow. Shallow water models have merely been applied to this kind of challenging problems. Bourdarias and Gerbi [98] applied a 1-D Godunov type model to bores running up partially pressurized pipes. Their model qualitatively reproduced the observed water surface profiles; however, they did not make comparison between the experimental and numerical celerity of the shock. Unami and Alam [49] demonstrated that the 1-D FEVM model reasonably simulates a hydraulic bore in a rectangular flume, accurately capturing the celerity of shock fronts.

A laboratory experiment in a $20 \mathrm{~m}$ long flume having a rectangular cross-section with the width of $0.60 \mathrm{~m}$ and the bed slope of 0.01 was carried out. Initially a uniform supercritical flow with the water depth of $0.026 \mathrm{~m}$ and the discharge of $12.95 \mathrm{~L} / \mathrm{s}$ was set up. The Froude number of the initial steady flow was 1.64. Downstream end of the flume was suddenly closed to generate a hydraulic bore propagating upstream. The front position of the shock was estimated from a video image as

$$
t=\left(0.2137 x^{*}+1.9344\right) x^{*} .
$$

where $x^{*}$ is the position of the front measured from the downstream end of the flume. The Manning's coefficient $n$ of the flume is determined as $0.0106 \mathrm{~s} / \mathrm{m}^{1 / 3}$ so that the same uniform supercritical flow is computationally set up. The entire flume is discretized into a computational mesh with $\mathrm{N}_{\mathrm{e}}=3,992$ elements and $\mathrm{N}_{\mathrm{n}}=2,255$ nodes. The time increment is set as $0.01 \mathrm{~s}$. The order of the computed line discharge $q$ at the initial steady flow $(t=0 \mathrm{~s})$ is smaller than $O\left(10^{-10}\right) \mathrm{m}^{2} / \mathrm{s}$ in the entire domain despite the used triangular mesh is not structured.

Figures 12 shows the computed water surface profiles at the interval of $10 \mathrm{~s}$. Observed positions of the shock are also plotted in the figure. The 2-D FEVM model adequately predicts the celerity of the shock with a slight underestimation, which is considered due to the hydrostatic pressure assumption of the 2-D SWEs. Similar results have been obtained for the 1-D FEVM model [49]. The computational results of this test problem show satisfactory accuracy of the 2-D FEVM model for transient transcritical flows involving moving shocks. 


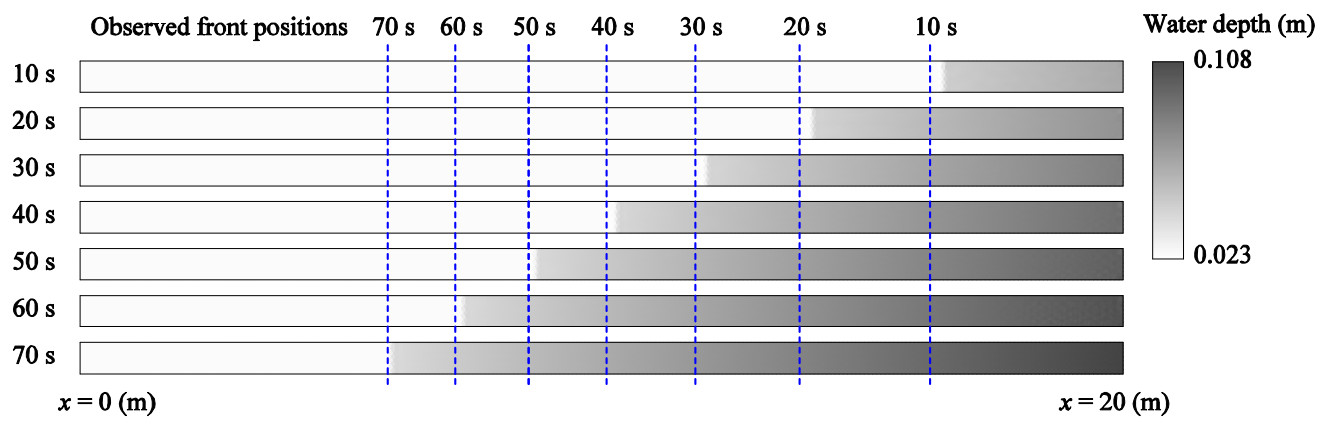

Figure 12 Computed water surface profiles for the hydraulic bore in a rectangular flume.

\subsection{Hydraulic bore in a rectangular flume with an obstacle}

Another hydraulic experiment was carried out utilizing the same rectangular flume in order to further validate the 2-D FEVM model. A bricks-made obstacle with the width of $0.208 \mathrm{~m}$ and the length of $0.816 \mathrm{~m}$ was placed in the flume. The downstream side of the obstacle was at $9.282 \mathrm{~m}$ from the downstream end of the flume. One of the long sides of the obstacle was along the centerline of the flume, creating a 0.300 m-width reach on the right side and a $0.092 \mathrm{~m}$-width reach on the left side. Consequently, an asymmetric multiply connected channel network with two junctions was created in the flume. A steady flow with the same inflow discharge of $12.95 \mathrm{~L} / \mathrm{s}$ was firstly set up. After that the downstream end of the flume was suddenly closed so that a hydraulic bore propagating upstream is created. Based on a video image, the arrival time of the hydraulic bore at the downstream end of the obstacle was estimated as $35.0 \mathrm{~s}$. In the experiment, water surface upstream of the obstacle developed an undular hydraulic jump due to the reflection effect by the channel separation. A very shallow water region was created just the downstream side of the obstacle followed up by oblique shocks. This test problem is therefore considered to serve as a critical test case to validate a 2-D model against complex transient transcritical flows. The Manning's coefficient $n$ for the domain is again set as $0.0105 \mathrm{~s} / \mathrm{m}^{1 / 3}$. The entire flume is discretized into a computational mesh with $\mathrm{N}_{\mathrm{e}}=9,353$ elements and $\mathrm{N}_{\mathrm{n}}=5,111$ nodes. A steady flow with the discharge of $12.95 \mathrm{~L} / \mathrm{s}$ is taken as the initial condition. The time increment is set as $0.005 \mathrm{~s}$.

Figure 13 shows the computed water surface profile in the entire flume at each time step. Figure 14 shows the computed water surface profile around the obstacle at each time step. The steady flow involves a hydraulic jump at the upstream of the obstacle and a very shallow water region with the depth of less than $0.010 \mathrm{~m}$ at immediately downstream of the obstacle, followed up by oblique shocks, all of them were observed in the experiment. As shown in Figure 13, the observed and computed front positions of the hydraulic jumps at the initial steady flow $(t=0 \mathrm{~s})$ agree well, which was not reasonably reproduced by the 1-D FEVM model. The undular nature of the hydraulic jump that the SWEs cannot deal with is considered to be handled using a Boussinesq type model [40, 99], but which is beyond the scope of this study. Figure 14 shows that the front of the hydraulic bore becomes gradually not perpendicular to the channel direction as it gets closer to the downstream end of obstacle, and approaches 
faster at the narrower left reach than the wider right reach. It has been confirmed that these computational results are consistent with the experimental observations. The arrival time of the hydraulic bore is computed as $34 \mathrm{~s}$ by the 1-D model and $36 \mathrm{~s}$ by the 2-D model; both of them are slightly different from the observed results mainly due to the hydrostatic pressure assumption of the SWEs. Nevertheless, the proposed 2-D model more adequately reproduces the general physical behaviors of the experimental results than the 1-D counterpart. The computational results show high capability of the 2-D FEVM model to handle complex transient transcritical flows with horizontally 2-D flow structures.

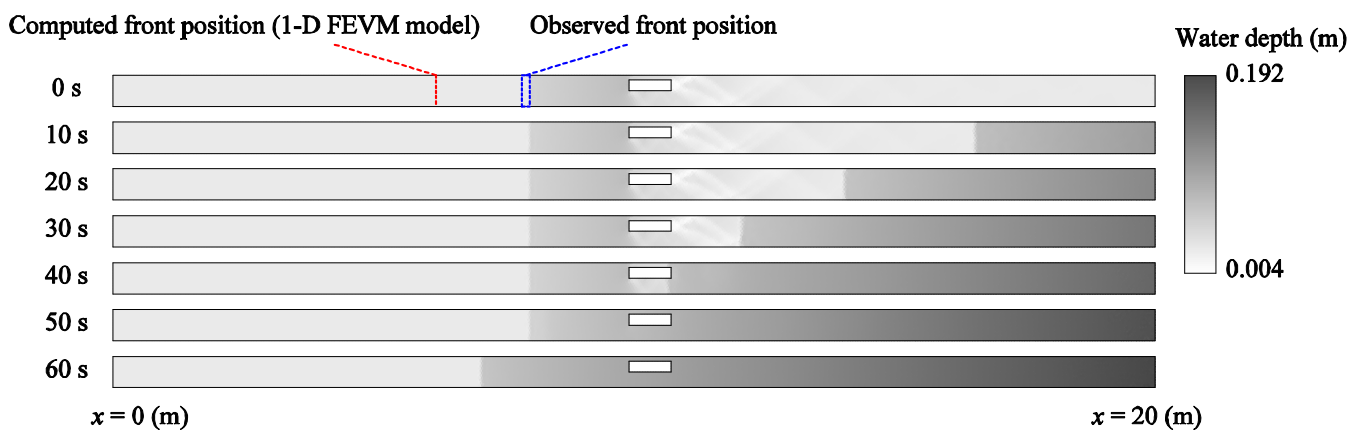

Figure 13 Computed water surface profiles for the hydraulic bore in a rectangular flume with an obstacle.
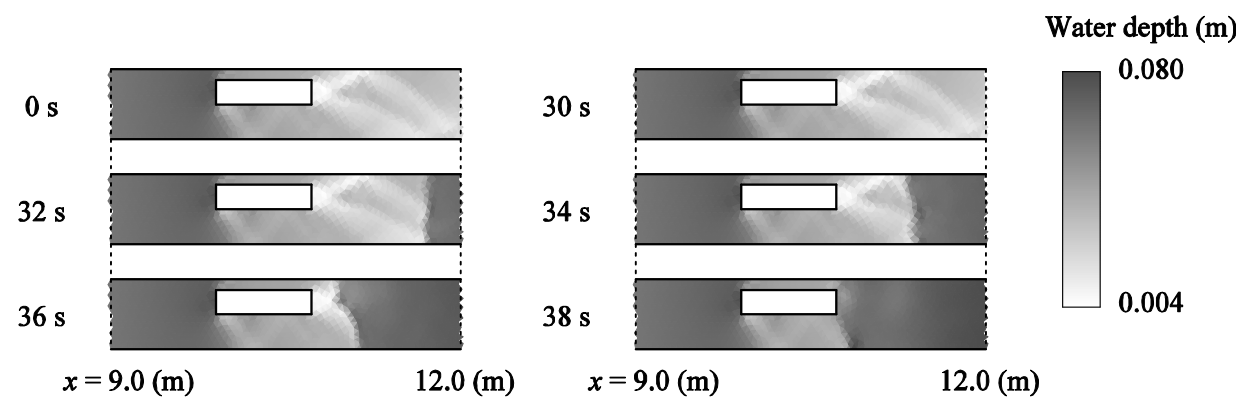

Figure 14 Computed water surface profiles around the obstacle.

\section{Conclusions}

A numerical model of the depth averaged horizontally 2-D SWEs utilizing both the FEM and FVM schemes, referred to as the 2-D FEVM model, was developed and verified through a series of test problems. The model does not use Riemann solvers and high-resolution algorithms, but uses a simple, heuristic numerical algorithm. The model utilizes the triangular mesh and applies the conforming, standard Galerkin FEM scheme to the continuity equation and the upwind, cell-centered FVM scheme to the momentum equations, respectively. Any special treatments for the gravitational source terms of the momentum equations were not employed in the model. The model applies a consistent, non-lumped spatial discretization to the temporal term of the continuity equation, whose numerical resolution in general requires the application of a linear system solver. This disadvantage was avoided by incorporating an efficient approximate matrix inversion method in the temporal integration, which does not rely on any linear system solvers. 
The computational results for the test problems indicated satisfactory accuracy of the 2-D FEVM model to a wide range of problems. The model computes slightly oscillatory solutions for the flows with shocks; nevertheless, the positions of the hydraulic jumps and the front positions of the hydraulic bores were quite well captured. The computational results for the last test problem, a hydraulic bore running up a rectangular flume with an obstacle, in particular indicated the limitations of the 1-D model and the superiority of the proposed 2-D model in simulating the complex transient transcritical flows.

Overall, it is concluded that the 2-D FEVM model is stable, sufficiently accurate, and versatile in spite of its simplicity. Improvement of the model to suppress non-physical oscillations around shocks will be achieved with a computationally efficient stabilization technique for the source terms in the momentum equations. Future research will focus on application of the 2-D FEVM model to real world problems with irregular bed topography and wet and dry interfaces through extensive analytical and numerical investigations.

\section{Acknowledgement}

This study is financially supported by the Japan Society for the Promotion of Science under grant $25 \square 2731$.

\section{References}

[1] Park IR, Kim KS, Kim J, Van SH. A volume-of-fluid method for incompressible free surface flows. International Journal for Numerical Methods in Fluids 2009; 61(12): 1582-1604.

[2] Yang C, Lin B, Jiang C, Liu Y. Predicting near-field dam-break flow and impact force using a 3D model. Journal of Hydraulic Research 2011; 48(6): 784-792.

[3] De Padova D, Mossa M, Sibilla S, Torti E. 3D SPH modelling of hydraulic jump in a very large channel. Journal of Hydraulic Research 2012; 51(2): 158-173.

[4] Coraci E, Umgiesser G, Zonta R. Hydrodynamic and sediment transport modelling in the canals of Venice (Italy). Estuarine, Coastal and Shelf Science 2007; 75(1-2): 250-260.

[5] Wahid SM, Babel MS, Bhuiyan AR. Hydrologic monitoring and analysis in the Sundarbans mangrove ecosystem, Bangladesh. Journal of Hydrology 2007; 332(3-4): 381-395.

[6] Guinot V. Multiple porosity shallow water models for macroscopic modelling of urban floods. Advances in Water Resources 2012; 37: 40-72.

[7] Liang Q, Borthwick AGL, Taylor PH. Wind-induced chaotic advection in shallow flow geometries. Part I: Circular basins. Journal of Hydraulic Research 2006a; 44(2): 170-179.

[8] Liang Q, Borthwick AGL, Taylor PH. Wind-induced chaotic advection in shallow flow geometries. Part II: Non-circular basins. Journal of Hydraulic Research 2006b; 44(2): 180-188.

[9] Stoker JJ. Water Waves: The Mathematical Theory with Applications. Wiley: U.S., 1958.

[10]Mclnerney D, Teubner M, Noye J. A second-order analytic solution for oscillatory wind-induced flow in an idealized shallow lake. Computers \& Fluids 2010; 39(2): 1500-1509. 
[11]Chen Y, Wu C, and Wang B. Similarity solution of dam-break flow on horizontal frictionless channel. Journal of Hydraulic Research 2012; 49(3): 384-387.

[12]Delestre O, Lucas C, Ksinant PA, Darboux F, Laguerre C, Vo TNT, James F, Cordier S. SWASHES: a compilation of shallow water analytic solutions for hydraulic and environmental studies. International Journal for Numerical Methods in Fluids 2013; 72(3): 269-320.

[13]Alcrudo F, Mulet J. Description of the Tous dam break case study (Spain). Journal of Hydraulic Research 2007; 45(Extra Issue): 45-57.

[14]Pilotti M, Maranzoni A, Tomirotti M, Valerio G. 1923 Gleno Dam break: Case study. Journal of Hydraulic Engineering 2011; 137(4): 480-492.

[15]Gabriella G, Natale L, Savi F, Velickovic M, Zech Y, Frazão SS. Flood wave propagation in steep mountain rivers. Journal of Hydroinformatics 2013; 15(1): 120-137.

[16] Trancoso AR, Braunschweig F, Leitao PC, Obermann M, Neves R. An advanced modeling tool for simulating complex river systems. Science of Total Environment 2009; 407(8): 3004-3016.

[17] Van Thang P, Chopard B, Lefèvre L, Ondo DA, Mendes E. Study of the 1D lattice Boltzmann shallow water equation and its coupling to build a canal network. Journal of Computational Physics 2012; 229(19): 7373-7400.

[18]Bhuiyan MJAN, Dutta D. Assessing impacts of sea level rise on river salinity in the Gorai river network, Bangladesh. Estuarine, Coastal and Shelf Science 2012; 96(1): 219-227.

[19]Horritt MS, Bates PD. Evaluation of 1D and 2D numerical models for predicting river flood inundation. Journal of Hydrology 2002; 268(1-4): 87-99.

[20]Neal J, Villanueva I, Wright N, Willis T, Fewtrell T, Bates P. How much physical complexity is needed to model flood inundation? Hydrological Processes 2012; 26(15): 2264-2282.

[21]Roe P. Approximate Riemann solvers, parameter vectors, and difference schemes. Journal of Computational Physics 1981; 43(2): 357-372.

[22] Osher S, Solomon F. Upwind difference schemes for hyperbolic systems of conservation laws. Mathematics of Computation 1982; 38(158): 339-374.

[23] Wu Y, Cheung KF. Explicit solutions to the exact Riemann problem and application in nonlinear shallow-water equations. International Journal for Numerical Methods in Fluids 2008; 57(11): 1649-1668.

[24] Toro EF, Garcia-Navarro P. Godunov-type methods for free-surface shallow water flows: A review. Journal of Hydraulic Research 2007; 45(6): 736-751.

[25] Van Leer B. Towards the ultimate conservative difference scheme II: Monotonicity and conservation combined in a second order scheme. Journal of Computational Physics 1974; 14(4): 361-370.

[26] Harten A, Lax P, Van Leer B. On upstream differencing and Godunov-type schemes for hyperbolic conservation laws. SIAM Review 1983; 25(1): 35-61.

[27]Rogers B, Fujihara M, Borthwick AGL. Adaptive Q-tree Godunov-type scheme for shallow water equations. International Journal for Numerical Methods in Fluids 2001; 35(3): 247-280.

[28]Zhou F, Chen G, Noelle S, Guo H. A well-balanced stable generalized Riemann problem scheme for shallow water equations using adaptive moving unstructured triangular meshes. International Journal for Numerical Methods in Fluids 2013; in 
press, 18pp. DOI: 10.1002/fld.3800

[29] Van Reeuwijk M. A mimetic mass, momentum and energy conserving discretization for the shallow water equations. Computers \& Fluids ds 2011; 46(1): 411-416.

[30] Van't Hof B, Veldman AEP. Mass, momentum and energy conserving (MaMEC) discretizations on general grids for the compressible Euler and shallow water equations. Journal of Computational Physics 2012; 231(14): 4723-4744.

[31] Shi Y, Ray RK, Nguyen KD. A projection method-based model with the exact C-property for shallow-water flows over dry and irregular bottom using unstructured finite-volume technique. Computers \& Fluids 2013; 76: 178-195.

[32]Delis AI, Nikolos IK, Kazolea M. Performance and comparison of cell-centered and node-centered unstructured finite volume discretizations for shallow water free surface flows. Archives of Computational Methods in Engineering 2011; 18(1): $57-118$.

[33] Mishev ID. Finite volume methods on Voronoi meshes. Numerical Methods for Partial Differential Equations 1998; 14(2): 193-212.

[34]Casulli V. A high-resolution wetting and drying algorithm for free surface hydrodynamics. International Journal for Numerical Methods in Fluids 2009; 60(4): 391-408.

[35] Stelling SG, Duinmeijer SPA. A staggered conservative scheme for every Froude number in rapidly varied shallow water flows. International Journal for Numerical Methods in Fluids 2012; 43(12): 1329-1354.

[36] Gallouët T, Gastaldo L, Herbin R, Latch JC. An unconditionally stable pressure correction scheme for the compressible barotropic Navier-Stokes equations. Mathematical Modelling and Numerical Analysis 2008; 42: 303-331.

[37]Boscheri W, Dumbser M, Righetti M, A semi-implicit scheme for 3D free surface flows with high-order velocity reconstruction on unstructured. International Journal for Numerical Methods in Fluids 2012; in press. DOI: 10.1002/fld.3753

[38] Vidović D, Segal A, Wesseling P. A superlinearly convergent Mach-uniform finite volume method for the Euler equations on staggered unstructured grids. Journal of Computational Physics 2006; 217(2): 277-294.

[39]Kazolea M, Delis AI, Nikolos IK, Synolakis CE. An unstructured finite volume numerical scheme for extended 2D Boussinesq-type equations. Coastal Engineering 2012; 69: 42-66.

[40] Ambrosi D, Corti S. Pennati V, Salcri F. Numerical simulation of unsteady flow at Po river delta. Journal of Hydraulic Engineering 1996; 122(12): 735-743.

[41] Unami K, Kawachi T, Babar MM, Itagaki H. Tow-dimensional numerical model of spillway flow. Journal of Hydraulic Engineering 1999; 125(4): 369-375.

[42]Idelsohn SR, Oñate E. Finite volumes and Finite elements: Two good friends. International Journal for Numerical Methods in Engineering 1994; 37(19): 3323-3341.

[43]Postma L, Hervouet JM. Compatibility between finite volumes and finite elements using solutions of shallow water equations for substance transport. International Journal for Numerical Methods in Fluids 2007; 53(9): 1495-1507.

[44]Akbar M, Aliabadi S. Hybrid numerical methods to solve shallow water equations for hurricane induced storm surge modeling. Environmental modeling and software 2013; in press, $11 \mathrm{pp}$.

[45]Mewis P. Sparse element for shallow water wave equations on triangle meshes. 
International Journal for Numerical Methods in Fluids 2013; 20pp. DOI: $10.1002 /$ fld.3761

[46] Wang JW., Liu RX. Combined finite volume-finite element method for shallow water equations. Computers \& Fluids 2005; 34(10): 1199-1222.

[47] Ishida K, Yangyuoru M, Unami K, Kawachi T, Application of shallow water equations to analyze runoff processes in hilly farmlands. Paddy and Water Environment 2011; 9(4): 393-401.

[48] Ishida K, Unami K, Kawachi T. Application of one-dimensional shallow water model to flows in open channels with bends. Journal of Rainwater Catchment Systems 2012; 17(2): 15-23.

[49]Unami K, Alam AHMB. Concurrent use of finite element and finite volume methods for shallow water equations in locally 1-D channel networks. International Journal for Numerical Methods in Fluids 2012; 69(2): 255-272.

[50] Szymkiewicz R. Numerical Modelling in Open Channel Hydraulics, Springer, 2010. DOI 10.1007/978-90-481-3674-2_8.

[51]Zarmehi F., Tavakoli A., Rahimpour M. On numerical stabilization in the solution of Saint-Venant equations using the finite element method. Computers and Mathematics with Applications 2011; 62(4): 1957-1968.

[52] Yoshioka H, Unami K, Fujihara M. On discretization of the one-dimensional shallow water numerical model. Proceedings of the Annual Meeting of Applied Hydraulics in 2012, pp. 96-100. (in Japanese)

[53] Yoshioka H, Unami K, Fujihara M. A dual grid based finite volume model for dam break flows in multiply connected open channel networks with general cross-sections. Proceedings of the $62^{\text {nd }}$ National Congress of Theoretical and Applied Mechanics, OS19-09; 2013: 2pp. (in Japanese)

[54]Hauke G. A stabilized finite element method for the Saint-Venant equations with application to irrigation. International Journal for Numerical Methods in Fluids 2002; 38(10): 963-984.

[55] Yazdi SSR, Kermani MZ. Numerical solution of tidal currents at marine waterways using wet and dry technique on Galerkin finite volume algorithm. Computers \& Fluids 2009; 38(10): 1876-1886.

[56] Takse S, Kashiyama K, Tanaka S, Tezduyar TE. Space-time SUPG formulation of the shallow-water equations. International Journal for Numerical Methods in Fluids 2010; 64(10-12): 1379-1394.

[57] Katopodes ND. A dissipative Galerkin scheme for open channel flow. Journal of Hydraulic Engineering 1984; 110(4): 450-466.

[58] Atallah M, Hazzab A. A Petrov-Galerkin scheme for modeling 1D channel flow with varying width and topography. Acta Mechanica 2013; 224(4): 707-725.

[59] Greenberg JM, Reroux AY. A well-balanced scheme for the numerical processing of source terms in hyperbolic equations. SIAM Journal of Numerical Analysis 1996; 33(1): 1-16.

[60]Pu JH, Cheng NS, Tan SK, Shao S. Source term treatment of SWEs using surface gradient upwind method. Journal of Hydraulic Research 2012; 50(2): 145-153.

[61] Touma R, Khankan S. Well-balanced unstaggered central schemes for one and two-dimensional shallow water equation systems. Applied Mathematics and Computation, 218(10), 5948-5960.

[62]Neisis J, Steinbach I. Finite element integration for the control volume method. 
Communications in Numerical Methods in Engineering 1996; 12(9): 543-555.

[63] Ciu H, Pietrzak JD, Stelling GS. A finite volume analogue of the $P_{1}^{N C}-P_{1}$ finite element: With accurate flooding and drying. Ocean Modelling 2012; 35(1-2): 16-30.

[64]Christon MA, Martinez MJ, Voth TE. Generalized Fourier analyses of the advection-diffusion equation-Part I: One-dimensional domains. International Journal for Numerical Methods in Fluids 2004; 45(8): 839-887.

[65] Voth TE, Martinez MJ, Christon MA. Generalized Fourier analyses of the advection-diffusion equation-Part II: Two-dimensional domains. International Journal for Numerical Methods in Fluids 2004; 45(8): 889-920.

[66] Selimin V. Finite element solution of hyperbolic equation I. One dimensional case. INRIA Research Report, 655, 1987; 29pp.

[67]Fang CC, Sheu TWH. Two element-by-element iterative solutions for shallow water equations. SIAM Journal of Scientific Computing 2001; 22(6): 2075-2092.

[68]Guermond JL, Pasuquetti R. A correction technique for the dispersive effects of mass lumping for transport problems. Journal of Numerical Methods in Applied Mechanics and Engineering 2013; 253(1): 186-198.

[69]Unami K, Kawachi T, Kranjac-Berisavljevic G, Abagale FK, Maeda S, Takeuchi J. Case study: Hydraulic modeling of runoff processes in Ghanaian inland valleys. Journal of Hydraulic Engineering 2009; 135(7): 539-553.

[70]Quarteroni A, Valli A. Numerical approximation of partial differential equations, Springer, 2008.

[71]Toro EF. Riemann solvers and numerical methods for fluid dynamics, Springer, 2010.

[72] Goutal N and Maurel F. Proceedings of the 2nd Workshop on Dam-break Wave Simulation, EDF-DER Report, HE-43/97/016/B, 1997.

[73]Fennema RJ, Chaudhry MH. Explicit method for 2-D transient free-surface flows. Journal of Hydraulic engineering 1991; 116(8): 1013-1034.

[74] Whitham GB. Linear and nonlinear waves, Wiley U.S., 1999.

[75] Meselhe EA and Holly Jr. FM. Invalidity of Preissmann scheme for transcritical flow. Journal of Hydraulic Engineering 1997; 123(7): 652-655.

[76] Audusse E, Bristeau MO. A well-balanced positivity preserving "second-order" scheme for shallow water flows on unstructured meshes. Journal of Computational Physics 2005; 206(1): 311-333.

[77]Akoh R, Ii S, Xiao F. A multi-moment finite volume formulation for shallow water equations on unstructured mesh. Journal of Computational Physics 2010; 229(12): 4567-4590.

[78]Liang D, Falconer RA, Lin B. Comparison between TVD-MacCormack and ADI-type solvers of the shallow water equations. Advances in Water Resources 2006; 29(12): 1833-1845.

[79] Anastasiou K, Chan CT. Solution of the 2D shallow water equations using the finite volume method on unstructured triangular method. International Journal for Numerical Methods in Fluids 1997; 24(11): 1225-1245.

[80] Mingham CG, Causon DM. High-resolution finite-volume method for shallow water flows. Journal of Hydraulic engineering 1998; 124(6): 605-614.

[81] Valiani A, Caleffi V, Zanni A. Finite volume scheme for 2D shallow-water equations. Application to the Malpasset dam-break. Proceedings of the $4^{\text {th }}$ 
Concerted Action on Dambreak Modelling Workshop, 1999, 63-94.

[82]Delis AI, Mathioudakis EN. A finite volume method parallelization for the simulation of free surface shallow water flows. Mathematics and Computers in Simulation 2009;79(11): 3339-3359

[83]Aliabadi S, Akbar M, Patel R. Hybrid finite element/volume method for shallow water equations. International Journal for Numerical Methods in Engineering 2010; 83(13): 1719-1738.

[84]Liu H, Zhou JG, Burrows R. Lattice Boltzmann simulations of the transient shallow water flows. Advances in Water Resources 2010; 33(4): 387-396.

[85] Li G, Lu C, Qiu J. Hybrid well-balanced WENO schemes with different indicators for shallow water equations. Journal of Scientific computing 2012; 51(3): 527-559.

[86] Ouyang C, He S, Xu Q, Luo Y, Zhang W, A MacCormack-TVD finite difference method to simulate the mass flow in mountainous terrain with variable computational domain. Computers and Geosciences 2013; 52: 1-10.

[87]Li G, Gao J, Liang Q. A well-balanced weighted essentially non-oscillatory scheme for pollutant transport in shallow water. International Journal for Numerical Methods in Fluids 2013; 71(12): 1566-1587.

[88]Delis AI, Katsaounis T. Numerical solution of the two-dimensional shallow water equations by the application of relaxation methods. Applied Mathematical Modelling 2005; 29(8): 754-783.

[89]Lin P, Wu Y, Bai J, Lin Q. A numerical study of dam-break flow and sediment transport from a quake lake. Journal of Earthquake and Tsunami 2010; 5(5): 401-428.

[90]Zoppou C, Roberts S. Numerical solution of the two-dimensional unsteady dam break. Applied Mathematical Modelling 2000; 24(7): 457-475.

[91] Yekta AHA, Banihashem MA. A Godunov-type fractional semi-implicit method based on staggered grid for dam-breaking modeling. International Journal for Numerical Methods in Fluids 2011; 67(10): 1291-1309.

[92] Alcrudo F, Garcia-Navarro P. A high-resolution Godunov-type scheme in finite volumes for the 2D shallow water equations. International Journal for Numerical Methods in Fluids 1993; 16(6): 489-505.

[93]Tseng MH. Explicit finite volume non-oscillatory schemes for 2D transient free-surface flows. International Journal for Numerical Methods in Fluids 1999; 30(7): 831-843.

[94]Lin GF, Lai JS, Guo WD. Finite volume component-wise TVD schemes for 2D shallow water equations. Advances in Water Resources 2003; 26(8): 861-873.

[95] Yoon TH, Kang SK. Finite volume model for two-dimensional shallow water flows on unstructured grids. Journal of Hydraulic Engineering 2004; 130(7): 678-688.

[96] Wang JW, Liu RX. A comparative study of finite volume methods on unstructured meshes for simulation of 2D shallow water wave problems. Mathematics and Computers in simulation 2000; 53(3), 171-184.

[97] Valiani A, Caleffi V. Brief analysis of shallow water equations suitability to numerically simulate supercritical flows in sharp bends. Journal of Hydraulic Engineering 2005; 131(10): 912-916.

[98]Bourdarias C, Gerbi S. A finite volume scheme for a model coupling free surface and pressurised flows in pipes. Journal of computational and Applied Mechanics 2007; 207(1), 109-131. 
[99]Kim DH, Lynett PJ. Dispersive and nonhydrostatic pressure effects at the front of surge. Journal of Hydraulic Engineering 2011; 137(7): 754-765. 